\title{
Studying the formation process of clusters in solution at the initial stage of protein crystallization
}

\author{
Bing Xiong \\ School of Materials Science and \\ Engineering, \\ Anhui University of Technology, \\ Maanshan, China \\ xb4512112@126.com
}

\author{
Guoliang Dai* \\ Key Laboratory of Microgravity \\ (National Microgravity Laboratory), \\ Institute of Mechanics, Chinese \\ Academy of Sciences, \\ Beijing, China \\ dspr@imech.ac.cn
}

\author{
Weihuo Li \\ School of Materials Science and \\ Engineering, \\ Anhui University of Technology, \\ Maanshan, China \\ whli@ahut.edu.cn
}

\begin{abstract}
The monomers or aggregates of protein molecules in protein crystallization solution at the initial stage of crystallization process are the basis of subsequent nucleation process. Tanaka et al (Tanaka S., et al, J. Cryst. Growth, 1996) concluded that there are units and clusters of lysozyme molecules in solution at the beginning of lysozyme crystallization process, and the decrease of clusters' amount showed the nucleation process comes up. Therefore, cluster of protein molecules in solution is one of important parameters, by which we can judge the crystallization process, in particular, nucleation process. However, the formation process of lysozyme clusters was not reported up to now. In this paper, we studied the aggregates of lysozyme molecules in solution 1 hr and 12 hrs after the mixture of lysozyme and $\mathrm{NaCl}$ (precipitant) solution, by atomic force microscopy (AFM) and dynamic light scattering (DLS). Moreover, we studied the effect of solution temperature on the aggregates in solution. Our results showed that, $1 \mathrm{hr}$ after the mixture of lysozyme and $\mathrm{NaCl}$ solution, the mean size of aggregates in solution first increased and then decreased. Our results also showed that the aggregates of lysozyme molecules in solution gradually disassembled and clusters of lysozyme molecules appeared within 12 hrs after the mixture of lysozyme and $\mathrm{NaCl}$ solution. The decrease of solution temperature decreased the period for appearance of clusters of lysozyme molecules in solution.
\end{abstract}

Keywords-protein crystallization; aggregate; unit; cluster

\section{INTRODUCTION}

Has been a goal of Crystal Growth workers looking for the optimization of protein crystal growth conditions, but the growth of protein crystals in a way, "science + experience", different proteins have different growth conditions, it is difficult to some sort of completely adapted to all protein crystal growth conditions and determine crystal whether the growth standards.

Protein crystal growth can be roughly divided into nucleation, nucleation, crystal and raised such as three-phase [1] At present, due to lack of research tools and other reasons, protein crystal growth research work focuses on the nucleation and crystal in two stages and grew up, etc. ${ }^{[2]}$, Few reports on the preliminary study of the nucleation. Prenucleation protein growth in the growth solution element and the corresponding aggregates of the state (such as: size, morphology and so on) change will directly affect the nucleation ${ }^{[2]}$. This is due to the aggregation which can not crystallize emerged mass-fractal clusters ${ }^{[3]}$, but in grow crystals of protein in the growth solution, the disordered state of the aggregation would turn into the ordered state clusters

${ }^{[4,5]}$.Therefore, the research of the initial stage of growth of protein and clusters gr wth formation process in solution will help to analyze the emergence of clusters body condition and provide appropriate conditions for protein crystal growth. In addition, protein crystal growth is closely related to precipitant types and concentration, temperature and other parameters, Aggregates state changes and the formation of clusters corresponding with the precipitant, temperature and other parameters chang ${ }^{[6]}$. Therefore, to study formation of clusters under the conditions of the parameters change, and also contribute to screening the unknown crystal growth conditions such as protein precipitant, temperature and other parameters.

\section{MATERIALS AND METHODS}

\section{A. Reagents}

Chemical reagents used in the experiment are AR. the reagents used by the light scattering experiments before use were centrifuged for $30 \mathrm{~min}(13,000 \mathrm{r} / \mathrm{min})$, and then filtered $0.22 \mu \mathrm{m}$ micro porous membrane (Millipore Corporation, USA), to remove dust as possible.

Hen egg white lysozyme ( NO.: L6876) Purchased from Sigma (USA) (by three times recrystallization), without further processing. Buffer solution contained different concentrations of $\mathrm{NaCl}$ and $0.1 \mathrm{~mol} / \mathrm{L} \mathrm{HAc}-\mathrm{NaAc}, \mathrm{pH} 4.5$.

Light scattering (BI-200SM, Brookhaven Instruments, Inc.) light source wavelength of $488 \mathrm{~nm} \mathrm{Ar}^{+}$laser (Billion Austrian laser, $150 \mathrm{~mW})$. LTD6G-type thermostatic circulator (UK Grant Instruments, Inc.). Light scattering sample cell made of optical glass, the underside is a square, side length of $5 \mathrm{~mm}$, high $75 \mathrm{~mm}$, wall thickness of $1 \mathrm{~mm}$. Production of atomic force microscopy and Ultralevers 20 cantilever by Park Scientific Instrument Company.

\section{B. Dynamic Light Scattering (DLS)}

DLS works with a fixed scattering angle of $90^{\circ}$. Decalin in the scattering chamber and the sample solution was contanted temperature by an external constant temperature circulator. Deal with the calculation software that came with the dynamic light scattering. Calculation method using the NNLS (non-negatively constrained least squares: multiple 
pass). DLS experimental results presented in the $\mathrm{X}$-axis using logarithmic coordinates, reflecting the scattering of the particle diameter distribution, the $\mathrm{Y}$-axis reflects the intensity of the absolute value, but of the particle size of particles scattering light intensity percentage (used strongest scattering light intensity as $100 \%$ ).

\section{Atomic Force Microscopy (AFM)}

AFM observes the size and morphology of the aggregates adsorbed on the silicon surface. The approach of silicon: silicon soak in solution which contain $30 \% \mathrm{H}_{2} \mathrm{O}_{2}, 70 \%$ $\mathrm{H}_{2} \mathrm{SO}_{4}(\mathrm{~V} / \mathrm{V})$ for $20 \mathrm{~min}$, then washed with deionized water. Save the prepared silicon chip in deionized water, dry with nitrogen before use it. Prepared silicon chips are used within an hour. The experiment will be a certain concentration of the protein solution drip onto the silicon surface, waiting for a certain period of time (the choice of the adsorption time is based on a series of adsorption experiments to determine), washed with deionized water and then evaporated to dryness. Using tapping Mode and Ultralevers20 micro cantilever, the experimental results was obtained at room temperature at 20 , Humidity $50 \sim 60 \%$. Experimental data processed by the instrument software (Image Browser Version 1.0.11), then you can get the size and height of the aggregates.

\section{RESULTS AND DISCUSSION}

\section{A. One-hour Change of the Aggregates in Lysozyme Solution}

Macromolecular crystallization process is the aggregation of disordered macromolecular aggregates in solution and then re-arranged in orderly clusters. Tanaka ${ }^{[5]}$ found that the lysozyme solution prior to crystallization, there are two aggregates, a small growth units, and the other is in large clusters, growth units composed of several lysozyme molecules, a size of about a few $\mathrm{nm}$. The size of clusters about $100 \mathrm{~nm} \sim 1 \mu \mathrm{m}$ 。At the beginning of aggregation, the number density of units decreased, the number density of clusters increased, which is the stage of units to the formation of clusters. The next stage, the number density of units is constant, the number density of clusters decreased, which means that the growth units to stop the aggregation of clusters body, While the aggregation between the clusters and clusters. But Tanaka ${ }^{[5]}$ hasn't studied the change of the clusters mean while the clusters formation process by growth time and make a quantitative research. We studied the changes of aggregates the protein solution and precipitant $(\mathrm{NaCl})$ solution on lysozyme crystal growth conditions within one hour by AFM and DLS.

At 20 , we added the precipitant solution $(7 \%$ (w / w) $\mathrm{NaCl}$ solution) into $40 \mathrm{mg} / \mathrm{ml}$ lysozyme solution, then the growth of solution within one hour after mixing were sampled (sampled once every 6 min for a total of 10 times), and the solution adsorbed by silicon for $5 \mathrm{~min}$, then the silicon observeing by AFM. Fig. 1 is the AFM results.
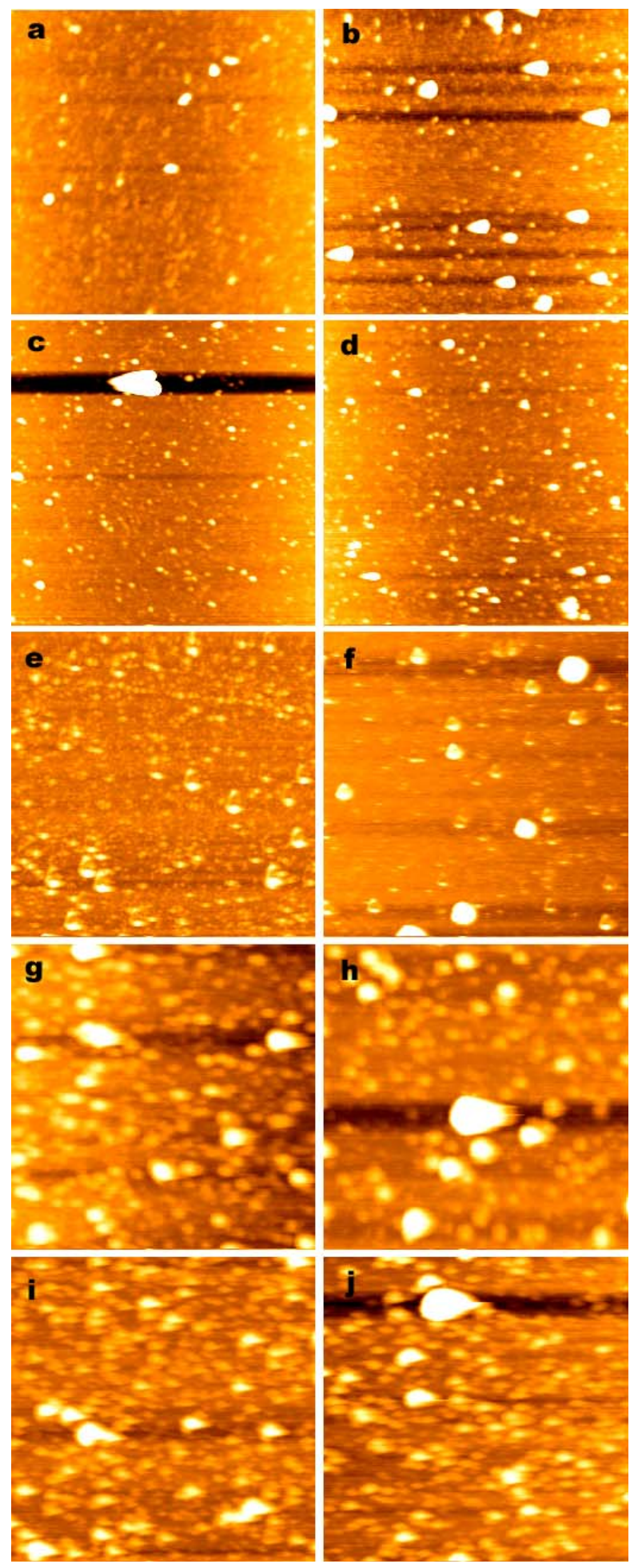

Fig.1 AFM results of lysozyme solution and precipitant solution within one hour after mixing, the solution after adsorption by silicon. Lysozyme concentration: $40 \mathrm{mg} / \mathrm{ml}$ precipitant solution: $7 \%(\mathrm{w} / \mathrm{w}) \mathrm{NaCl}$. AFM images of the scan range $2 \mu \mathrm{m} \times 2 \mu \mathrm{m}$ 。 Figure $\mathrm{a} \sim \mathrm{j}$ corresponding to the mixed solution for $6 \mathrm{~min}, 12 \mathrm{~min}, 18 \mathrm{~min}, 24 \mathrm{~min}, 30 \mathrm{~min}$, $36 \mathrm{~min}, 42 \mathrm{~min}, 48 \mathrm{~min}, 54 \mathrm{~min}, 60 \mathrm{~min}$. 
Fig.1, most graph emeraged special large aggregates, but if statistics the average size of aggregates, During the lysozyme solution with $\mathrm{NaCl}$ solution mixed 1 hour, The average size of aggregates became the first smaller and then larger (50 min or less), then the average size showing a decreasing trend (from $50 \mathrm{~min}$ to $60 \mathrm{~min}$ ). This shows that when $\mathrm{NaCl}$ solution was added to the lysozyme solution, the lysozyme molecules in solution occurrence aggregation at first, and the size of aggregates gradually increases, after 50 min, the aggregates began disaggregated.

The AFM observation is the results of the solution adsorbed to the silicon, may be the size of the aggregates in the solution made some changes ${ }^{[7,8]}$. Dynamic Light Scattering (DLS), mainly available to the hydrodynamic radius and the distribution of the particles in solution, and with the advantages of real-time and non-destructive observation $^{[9]}$ 。 Therefore, we took advantage of the DLS observed on the size of the aggregates which formed in the lysozyme solution and precipitant solution mixed solution within 1 hour. Experimental conditions and the observation time interval is the same with the AFM experiments. DLS results were shown in Fig. 2. The two sets of curves in Figure 2 is a parallel experiment. The average size of the aggregates become larger in the mixture solution during 45 min, the peak appeared at about $45 \mathrm{~min}$, and then began to decrease. DLS results with the results of the AFM is consistent with the overall size of the aggregates are showing the first increase and then a decreasing trend. The difference is that AFM results show that turning point appeared at 50 min but the results of the DLS is $45 \mathrm{~min}$. This difference may come from different measurement methods. DLS is a real-time observation, But AFM results is the result of solution adsorbed on the silicon chip for $5 \mathrm{~min}$, So the AFM results has a few minutes lag compared with real-time observation.

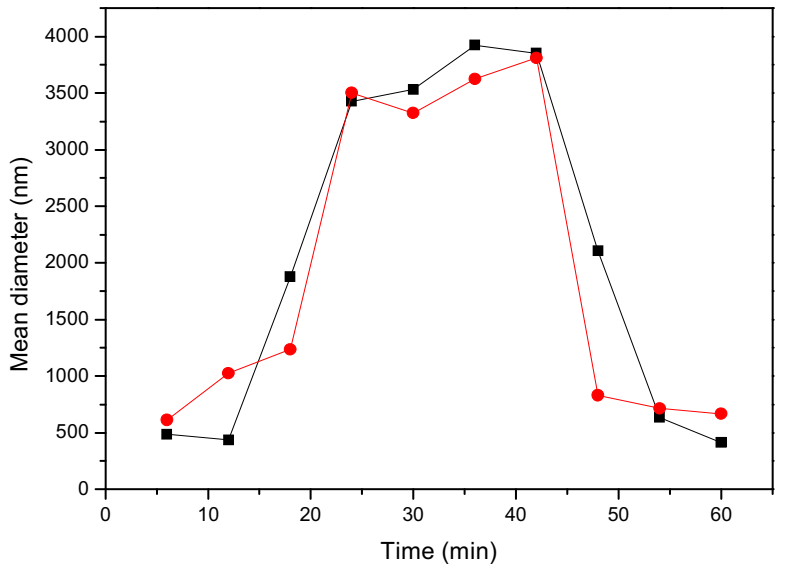

Fig. 2 The average size of the aggregates in solution changes with time in mixture solution contained lysozyme solution and precipitant solution within one hour. Lysozyme concentration: $40 \mathrm{mg} / \mathrm{ml}$, precipitant solution :7\% ( $w / w)$ $\mathrm{NaCl}$.

\section{B. Aggregates changes in lysozyme solution in 12 hours}

The previous AFM and DLS experimental results show that the size of the aggregates in the lysozyme solution grew with increase and then decrease trend in one hour. How is the change of the aggregates size after one hour, whether the aggregates began disaggregated step by step as Tanaka et $\mathrm{al}^{[5]}$ described clusters? Therefore, we studied and observed the changes of the aggregates in lysozyme solution after one hour by AFM. Within 12 hours after mixed the lysozyme solution and $\mathrm{NaCl}$ solution, the lysozyme solution sampled once every two hours, Aggregate size of the sample solution which adsorbed on the silicon was observed by AFM. The concentration lysozyme solution and $\mathrm{NaCl}$ solution is the same as previously. AFM results were shown in Fig. 3.
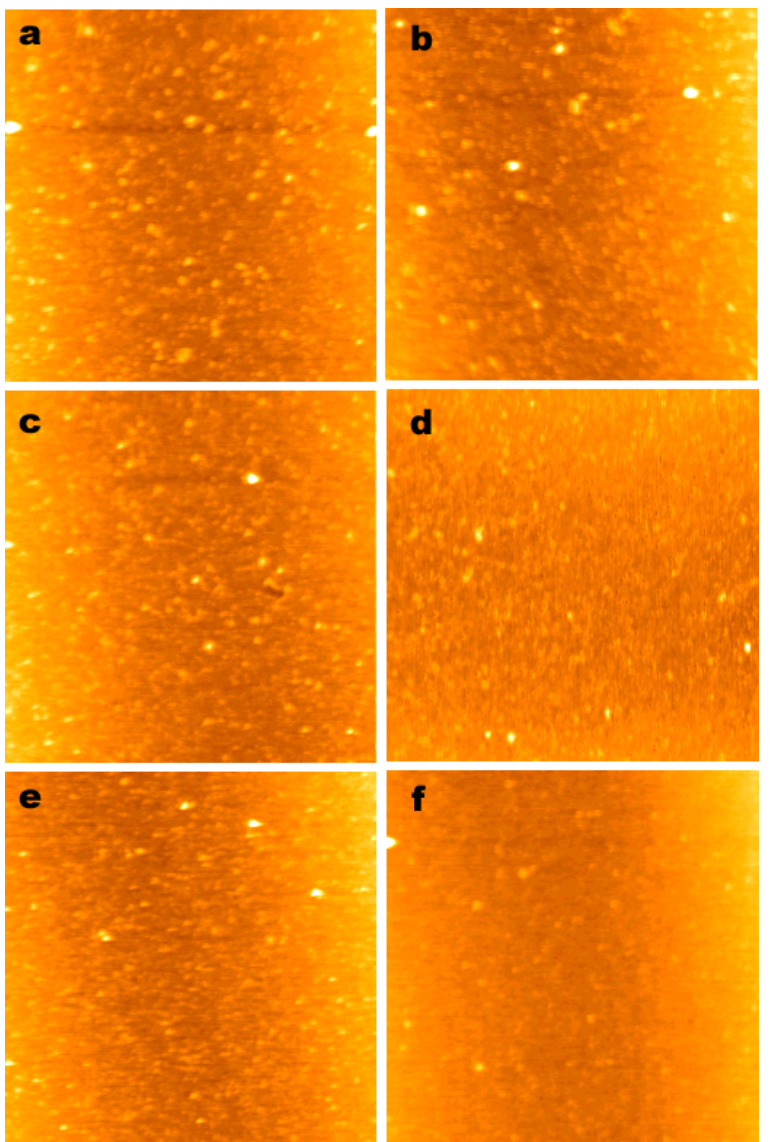

Fig.3 Within 12 hours after mixed the lysozyme solution and $\mathrm{NaCl}$ solution, the AFM results of the solution which adsorbed on the silicon. Lysozyme concentration: $40 \mathrm{mg} / \mathrm{ml}$, precipitant solution :7\% $(w / w) \mathrm{NaCl}$. AFM images of the scan range $2 \mu \mathrm{m} \times 2 \mu \mathrm{m}$. Figure $\mathrm{a} \sim \mathrm{j}$ corresponding to the mixed solution for $2 \mathrm{~h}, 4 \mathrm{~h}, 6 \mathrm{~h}, 8 \mathrm{~h}, 10$ and $12 \mathrm{~h}$.

Fig. 3 results showed that in our experimental conditions, when the mixed $\mathrm{NaCl}$ solution with lysozyme solution, the size of the aggregates in the crystal growth solution of lysozyme showing progressively smaller trend. After mixing 12 hours, the solution has been basically no large aggregates (Fig.3f) . Since lysozyme crystal nucleation required for approximately 12 hours under the conditions of crystal 
growth in lysozyme solution we used ${ }^{[10]}$. Therefore, Fig.3 showed that aggregates were almost disaggregated as clusters as Tanaka ${ }^{[5]}$ discribled. So that the growth solution from an early stage into the early stages of nucleation, and prepared the formation of nuclei.

\section{The effect of temperature on aggregates in lysozyme solution}

When the conditions are the same as in other growth soltion, the temperature change will make the protein saturation changes, thus changing the rate of protein crystal growth. There are no reports about the impact on the aggregates formation at the initial growth stage by temperature. The mixed lysozyme and $\mathrm{NaCl}$ solution was observed by DLS after mixed 8 hours. The experimental conditions is the same as previously, set the initial temperature at 20 , and then gradually cooling to $18{ }^{\circ} \mathrm{C}$, $17{ }^{\circ} \mathrm{C}, 16{ }^{\circ} \mathrm{C}, 15{ }^{\circ} \mathrm{C}$. DLS results were shown in Fig. 4. Fig. 4 results showed that when the temperature ranged 20 and 16 , the solution always has two parts of the aggregates. One part of aggregates in the average size approximately few nanometers, shows that this part of the aggregates is the same as an unit which Tanaka ${ }^{[5]}$ described. Another part of the aggregates are in the average size of $10 \mathrm{~nm}$ to $100 \mathrm{~nm}$. This value is much smaller than the average size which exited in 20 solution after the solution mixed one hour ( $500 \mathrm{~nm}$ ) (Fig. 2). Compared with Fig.2, we can conclude that after the disaggregated process was still continued after mixed the solution one hour, so the average size of aggregates is reduced to $10 \mathrm{~nm}$ to $100 \mathrm{~nm}$ after 8 hours. The size of aggregates is $100 \mathrm{~nm} \sim 1 \mu \mathrm{m}$ by Tanaka ${ }^{[5]}$, reports. Therefore, We believe that when the solution temperature is $20 \sim 16$, the aggregates in the solution close to the transition cluster body after eight hours. The results of Fig. 4 also shows that when the solution temperature dropped to $15^{\circ} \mathrm{C}$, the solution besides the above two parts of aggregates, emerging another aggregates which the average size of about $200 \mathrm{~nm} \sim 500 \mathrm{~nm}$. Since the solution mixed 8 hours at 15 , there are both aggregates and clusters in solution, it demonstrates That cluster formation process has already begun.

According to Fig.3, at 20 the clusters emerged at 12 hours. This indicates that lysozyme solution saturation increases with decreasing temperature, the time required to form clusters is reduced, this is consistent with that the supersaturation increase will reduce the time of growth of protein crystals.

\section{CONCLUSION}

Through our research, the results show that the size of aggregates in the solution within 1 hour firstly increases and then decreases. Within 12 hours, the aggregates in solution solution gradually gathered as clusters body. The cooling study results show that with the lower growth temperature of the solution, the solution reduced the time required to form cluster body.
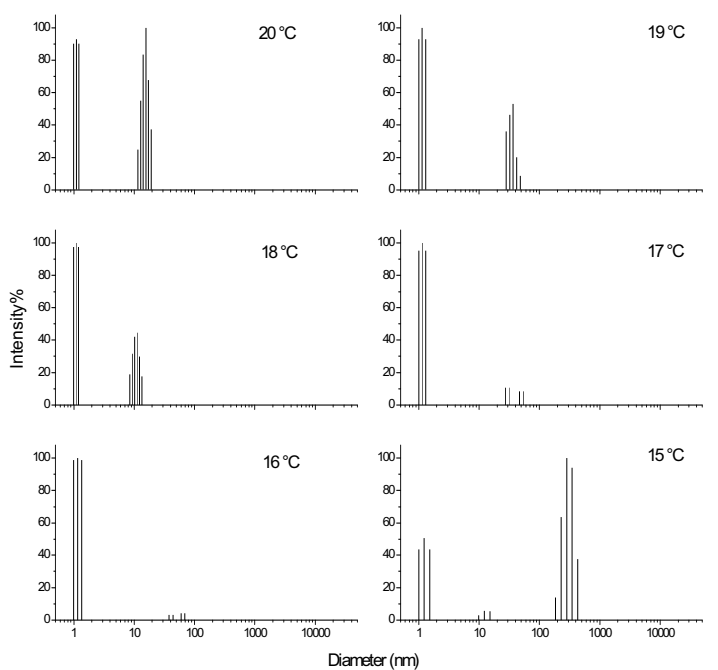

Fig. 4 The average size of the aggregates in solution changes with time in mixture solution contained lysozyme solution

and precipitant solution within 8 hours. Lysozyme

concentration: $40 \mathrm{mg} / \mathrm{ml}$, precipitant solution :7\% ( $w / w)$ $\mathrm{NaCl}$.

\section{ACKNOWLEDGMENT}

Thanks grant from National Basic Research Program of China (No. 2011CB710901) and Knowledge Innovation Program of the Chinese Academy of Sciences (No.KJCX2YW-L08)

\section{REFERENCES}

[1] Weber P.. In Advances in Protein Chemistry, Afinsen C B, Richards F M, Edsal J T, et al, eds. Vol. 41, New York, Academic Press, 1991, p.63

[2] Igarashi K., Azuma M., Kato J., et al. The initial stage of crystallization of lysozyme: a differential scanning calorimetric (DSC) study. Journal of Crystal Growth ,vol 204(1-2), 1999: pp.191-200

[3] Narayanan J., Liu X. Y.. Protein interactions in undersaturated and supersaturated solutions: a study using light and X-Ray Scattering. Biophysical Journal, vol 84(1), pp.523-532,2003

[4] Retailleau P., Ducruix A., Ries-Kautt M.. Acta Crystallographica Section D-Biological Crystallography: vol 58(1), pp.1576-1581, 2002

[5] Tanaka S., Yamamoto M., Kawashima K., et al. Kinetic study on the early stage of the crystallization process of two forms of lysozyme crystals by photon correlation spectroscopy, Journal of Crystal Growth: vol 168(1-4), pp.44-49,1996

[6] Dai G. L., Yu Y., Kang Q.. Study of the aggregates in lysozyme solution before crystal growth (In Chinese): Acta Chimica Sinica, vol 62(8), pp.757-761, 2004

[7] Willem N., Charles A. H.. In Proteins at Interfaces II: Fundamentals and Applications, Hhomas A H, John L B, eds. American Chemical Society, Washington. DC , 1995 , p.27

[8] Georgalis, Y, Schueler, J, Frank, J, et al. Advance in Colliod and Interface Science, vol 58(1), pp.57-86, 1995

[9] Poznanski J, Szymanski J, Basinska T, et al. Aggregation of aqueous lysozyme solutions followed by dynamic light scattering and $1 \mathrm{H}$ NMR spectroscopy. Journal of Molecular Liquids, vol 121(1), pp.21-26, 2005 